\title{
Jointly Embedding Entities and Text with Distant Supervision
}

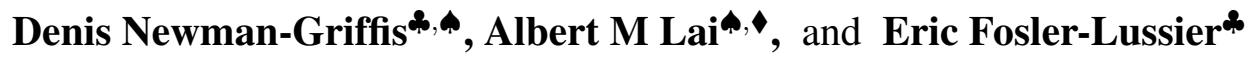 \\ *Department of Computer Science and Engineering, The Ohio State University, Columbus, $\mathrm{OH}$ \\ -Rehabilitation Medicine Department, Clinical Center, National Institutes of Health, Bethesda, MD \\ 'Institute for Informatics, Washington University in St. Louis, St. Louis, MO \\ \{newman-griffis.1, fosler-lussier.1\}@osu.edu amlai@wustl.edu
}

\begin{abstract}
Learning representations for knowledge base entities and concepts is becoming increasingly important for NLP applications. However, recent entity embedding methods have relied on structured resources that are expensive to create for new domains and corpora. We present a distantly-supervised method for jointly learning embeddings of entities and text from an unnanotated corpus, using only a list of mappings between entities and surface forms. We learn embeddings from open-domain and biomedical corpora, and compare against prior methods that rely on human-annotated text or large knowledge graph structure. Our embeddings capture entity similarity and relatedness better than prior work, both in existing biomedical datasets and a new Wikipedia-based dataset that we release to the community. Results on analogy completion and entity sense disambiguation indicate that entities and words capture complementary information that can be effectively combined for downstream use.
\end{abstract}

\section{Introduction}

Distributed representations of knowledge base entities and concepts have become key elements of many recent NLP systems, for applications from document ranking (Jimeno-Yepes and Berlanga, 2015) and knowledge base completion (Toutanova et al., 2015) to clinical diagnosis code prediction (Choi et al., 2016a,b). These works have taken two broad tacks for the challenge of learning to represent entities, each of which may have multiple unique surface forms in text. Knowledge-based approaches learn entity representations based on the structure of a large knowledge base, often augmented by annotated text resources (Yamada et al., 2016; Cao et al., 2017). Other methods utilize explicitly annotated data, and have been more popular in the biomedical domain (Choi et al., 2016a; Mencia et al., 2016). Both approaches, however, are often limited by ignoring some or most of the available textual information. Furthermore, such rich structures and annotations are lacking for many specialized domains, and can be prohibitively expensive to obtain.

We propose a fully text-based method for jointly learning representations of words, the surface forms of entities, and the entities themselves, from an unannotated text corpus. We use distant supervision from a terminology, which maps entities to known surface forms. We augment the well-known log-linear skip-gram model (Mikolov et al., 2013) with additional term- and entity-based objectives, and evaluate our learned embeddings in both intrinsic and extrinsic settings.

Our joint embeddings clearly outperform prior entity embedding methods on similarity and relatedness evaluations. Entity and word embeddings capture complementary information, yielding improved performance when they are combined. Analogy completion results further illustrate these differences, demonstrating that entities capture domain knowledge, while word embeddings capture morphological and lexical information. Finally, we see that an oracle combination of entity and text embeddings nearly matches a state of the art unsupervised method for biomedical word sense disambiguation that uses complex knowledge-based approaches. However, our embeddings show a significant drop in performance compared to prior work in a newswire disambiguation dataset, indicating that knowledge graph structure contains entity information that a purely text-based approach does not capture. 


\section{Related Work}

Knowledge-based approaches to entity representation are well-studied in recent literature. Several approaches have learned representations from knowledge graph structure alone (Grover and Leskovec, 2016; Yang et al., 2016; Wang et al., 2017). Wang et al. (2014), Yamada et al. (2016), and Cao et al. (2017) all use a joint embedding method, learning representations of text from a large corpus and entities from a knowledge graph; however, they rely on the disambiguated entity annotations in Wikipedia to align their models. Fang et al. (2016) investigate heuristic methods for joint embedding without annotated entity mentions, but still rely on graph structure for entity training.

The robust terminologies available in the biomedical domain have been instrumental to several recent annotation-based approaches. De Vine et al. (2014) use string matching heuristics to find possible occurrences of known biomedical concepts in literature abstracts, and use the sequence of these noisy concepts (without the document text) as input for skip-gram training. Choi et al. (2016c) and Choi et al. (2016a) use sequences of structured medical observations from patients' hospital stays for context-based learning. Finally, Mencia et al. (2016) take documents tagged with Medical Subject Heading (MeSH) topics, and use their texts to learn representations of the MeSH headers. These methods are able to draw on rich structured and semi-structured data from medical databases, but discard important textual information, and empirically are limited in the scope of the vocabularies they can embed.

\section{Methods}

In order to jointly learn entity and text representations from an unannotated corpus, we use distant supervision (Mintz et al., 2009) based on known terms, strings which can represent one or more entities. The mapping between terms and entities is many-to-many; for example, the same infection can be expressed as "cold" or "acute rhinitis", but "cold" can also describe the temperature or refer to chronic obstructive lung disease.

Mappings between terms and entities are defined by a terminology. ${ }^{1}$ We extracted terminologies from two well-known knowledge bases:

\footnotetext{
${ }^{1}$ Terminology is overloaded with both biomedical and lexical senses; we use it here strictly to mean a mapping between terms and entities.
}

\begin{tabular}{|c|c|c|}
\hline & UMLS & Wikipedia \\
\hline \# entities & $3,590,353$ & $9,723,785$ \\
\hline \# terms & $7,558,254$ & $17,147,756$ \\
\hline Max terms & 495 & 7,077 \\
\hline \multicolumn{3}{|c|}{ \# entities represented by $n$ terms } \\
\hline$n=1$ & $1,823,569(51 \%)$ & $6,828,958(70 \%)$ \\
\hline$n=2$ & $894,932(25 \%)$ & $1,565,109(16 \%)$ \\
\hline $3 \leq n \leq 10$ & $831,494(23 \%)$ & $1,143,452(12 \%)$ \\
\hline$n>10$ & $40,358(1 \%)$ & $186,266(2 \%)$ \\
\hline \multicolumn{3}{|c|}{ \# terms mapping to $n$ entities } \\
\hline$n=1$ & $7,473,902(98 \%)$ & $16,127,138(94 \%)$ \\
\hline$n=2$ & $69,816(1 \%)$ & $958,242(5 \%)$ \\
\hline $3 \leq n \leq 10$ & $14,366(<1 \%)$ & $62,062(<1 \%)$ \\
\hline$n>10$ & $170(\ll 1 \%)$ & $15(\ll 1 \%)$ \\
\hline
\end{tabular}

Table 1: Statistics of the many-to-many mapping between terms and entities in our terminologies, including the maximum \# of terms per entity.

The Unified Medical Language System (UMLS; Bodenreider, 2004); we use the mappings between concepts and strings in the MRCONSO table as our terminology. This yields 3.5 million entities, represented by 7.6 million strings in total.

Wikipedia; we use page titles and redirects as our terminology. This yields 9.7 million potential entities (pages), represented by 17.1 million total strings. Table 1 gives further statistics about the mapping between entities and surface forms in each of these terminologies.

While iterating through the training corpus, we identify any exact matches of the terms in our terminologies. ${ }^{2}$ We allow for overlapping terms: thus, "in New York City" will include an occurrence of both the terms "New York" and "New York City." Each matched term may refer to one or more entities; we do not use a disambiguation model in preprocessing, but rather assign a probability distribution over the possible entities.

\subsection{Model}

We extend the skip-gram model of Mikolov et al. (2013), to jointly learn vector representations of words, terms, and entities from shared textual contexts. For a given target word, term, or entity $v$, let $C_{v}=c_{-k} \ldots c_{k}$ be the observed contexts in a window of $k$ words to the left and right of $v$, and let $N_{v}=n_{-k, 1} \ldots n_{k, d}$ be the $d$ random negative samples for each context word. Then, the contextbased objective for training $v$ is

$$
O\left(v, C_{v}, N_{v}\right)=\sum_{c \in C_{v}} \log \sigma(\vec{c} \cdot \vec{v})+\sum_{n \in N_{v}} \log \sigma(-\vec{n} \cdot \vec{v})
$$

\footnotetext{
${ }^{2}$ We lowercase and strip special characters and punctuation from both terms and corpus text, and then find all exact matches for the terms.
} 


\begin{tabular}{l|c|c|c} 
& Pubmed & Wikipedia & Gigaword \\
\hline \# tokens & $2.6 \mathrm{~B}$ & $1.9 \mathrm{~B}$ & $4.3 \mathrm{~B}$ \\
\# mentions & $1.5 \mathrm{~B}$ & $1.4 \mathrm{~B}$ & $3.2 \mathrm{~B}$ \\
Avg $C P$ & 2.54 & 1.01 & 1.01 \\
\hline \multicolumn{5}{l}{ \% of entities by polysemy impact } \\
\hline$C P \geq 1$ & $99.1 \%$ & $98.6 \%$ & $98.8 \%$ \\
$C P \geq 2$ & $9.3 \%$ & $3.5 \%$ & $2.2 \%$ \\
$C P \geq 10$ & $0.3 \%$ & $0 \%$ & $\ll 0.1 \%$
\end{tabular}

Table 2: Statistics of our embedding training corpora. \# mentions is the number of exact matches found for terms in the relevant terminology. $\mathrm{CP}=$ corpus polysemy of a given entity. $\mathrm{B}=$ billion.

where $\sigma$ is the logistic function.

We use a sliding context window to iterate through our corpus. At each step, the word $w$ at the center of the window $C_{w}$ is updated using $O\left(w, C_{w}, N_{w}\right)$, where $N_{w}$ are the randomlyselected negative samples.

As terms are of variable token length, we treat each term $t$ as an atomic unit for training, and set $C_{t}$ to be the context words prior to the first token of the term and following the final token. Negative samples $N_{t}$ are sampled independently of $N_{w}$.

Finally, each term $t$ can represent a set of entities $E_{t}$. Vectors for these entities are updated using the same $C_{t}$ and $N_{t}$ from $t$. Since the entities are latent, we weight updates with uniform probability $\left|E_{t}\right|^{-1}$; attempts to learn this probability did not produce qualitatively different results from the uniform distribution. Thus, letting $T$ be the set of terms completed at $w$, the full objective function to maximize is:

$$
\begin{aligned}
\hat{O}= & O\left(w, C_{w}, N_{w}\right)+ \\
& \sum_{t \in T}\left[O\left(t, C_{t}, N_{t}\right)+\sum_{e \in E_{t}} \frac{1}{\left|E_{t}\right|} O\left(e, C_{t}, N_{t}\right)\right]
\end{aligned}
$$

Term and entity updates are only calculated when the final token of one or more terms is reached; word updates are applied at each step. To assign more weight to near contexts, we subsample the window size at each step from $[1, k]$.

\subsection{Training corpora}

We train embeddings on three corpora. For our biomedical embeddings, we use 2.6 billion tokens of biomedical abstract texts from the 2016 PubMed baseline (1.5 billion noisy annotations). For comparison to previous open-domain work, we use English Wikipedia (5.5 million articles from the 2018-01-20 dump); we also use the Gigaword 5 newswire corpus (Parker et al., 2011), which does not have gold entity annotations.
As our model does not include a disambiguation module for handling ambiguous term mentions, we also calculate the expected effect of polysemous terms on each entity that we embed using a given corpus. We call this the entity's corpus polysemy, and denote it with $C P(e)$. For entity $e$ with corresponding terms $T_{e}, C P(e)$ is given as

$$
C P(e)=\sum_{t \in T_{e}} \frac{f(t)}{Z} \operatorname{polysemy}(t)
$$

where $f(t)$ is the corpus frequency of term $t, Z$ is the frequency of all terms in $T_{e}$, and polysemy $(t)$ is the number of entities that $t$ can refer to.

Table 2 breaks down expected polysemy impact for each corpus. The vast majority of entities experience some polysemy effect in training, but very few have an average ambiguity per mention of $50 \%$ or greater. Most entities with high corpus polysemy are due to a few highly ambiguous generic strings, such as combinations and unknown. However, some specific terms are also high ambiguity: for example, Washington County refers to 30 different US counties.

\subsection{Hyperparameters}

For all of our embeddings, we used the following hyperparameter settings: a context window size of 2 , with 5 negative samples per word; initial learning rate of 0.05 with a linear decay over 10 iterations through the corpus; minimum frequency for both words and terms of 10 , and a subsampling coefficient for frequent words of 1e-5.

\subsection{Baselines}

We compare the words, terms, ${ }^{3}$ and entities learned in our model against two prior biomedical embedding methods, using pretrained embeddings from each. De Vine et al. (2014) use sequences of automatically identified ambiguous entities for skip-gram training, and Mencia et al. (2016) use texts of documents tagged with MeSH headers to represent the header codes. The most recent comparison method for Wikipedia entities is MPME (Cao et al., 2017), which uses link anchors and graph structure to augment textual contexts. We also include skip-gram vectors as a final baseline; for Pubmed, we use pretrained embeddings with optimized hyperparameters from Chiu et al. (2016a), and we train our own embeddings with word2vec for both Wikipedia and Gigaword.

\footnotetext{
${ }^{3}$ Unknown terms were handled by backing off to words.
} 


\begin{tabular}{|c|c|c|c|c|}
\hline \multirow[b]{2}{*}{ Method } & \multicolumn{2}{|c|}{ Full } & \multicolumn{2}{|c|}{ Filtered } \\
\hline & Sim & Rel & Sim & Rel \\
\hline \multicolumn{5}{|l|}{ Prior work } \\
\hline word2vec & 0.559 & 0.496 & & \\
\hline DeVine'14 & 0.455 & 0.422 & 0.534 & 0.482 \\
\hline Mencia'16 & 0.565 & 0.534 & 0.573 & 0.536 \\
\hline \multicolumn{5}{|l|}{ Proposed } \\
\hline Word & 0.561 & 0.490 & & \\
\hline Term & 0.619 & $0.557 *$ & & \\
\hline Entity & $0.633 *$ & $0.563 *$ & $0.614 *$ & $0.567 *$ \\
\hline Entity+Word & $0.653 *$ & $0.586 *$ & $0.615^{*}$ & $0.583 *$ \\
\hline+ Cross & $0.662 *$ & 0.588* & $0.622 *$ & $0.573 *$ \\
\hline
\end{tabular}

Table 3: Spearman's $\rho$ for similarity/relatedness predictions in UMNSRS. Filtered results indicate performance on the shared-vocabulary subset. *=significantly better $(p<0.05)$ than word baseline (full), DeVine et al (filtered).

\section{Evaluations}

Following Chiu et al. (2016b), Cao et al. (2017), and others, we evaluate our embeddings on both intrinsic and extrinsic tasks. To evaluate the semantic organization of the space, we use the standard intrinsic evaluations of similarity and relatedness and analogy completion. To explore the applicability of our embeddings to downstream applications, we apply them to named entity disambiguation. Results and analyses for each experiment are discussed in the following subsections.

\subsection{Similarity and relatedness}

We evaluate our biomedical embeddings on the UMNSRS datasets (Pakhomov et al., 2010), consisting of pairs of UMLS concepts with judgments of similarity (566 pairs) and relatedness (587 pairs), as assigned by medical experts. For evaluating our Wikipedia entity embeddings, we created WikiSRS, a novel dataset of similarity and relatedness judgments of paired Wikipedia entities (people, places, and organizations), as assigned by Amazon Mechanical Turk workers. We followed the design procedure of Pakhomov et al. (2010) and produced 688 pairs each of similarity and relatedness judgments; for further details on our released dataset, please see the Appendix.

For each labeled entity pair, we calculated the cosine similarity of their embeddings, and ranked the pairs in order of descending similarity. We report Spearman's $\rho$ on these rankings as compared to the ranked human judgments: Table 3 shows results for UMNSRS, and Table 4 for WikiSRS.

As the dataset includes both string and disambiguated entity forms for each pair, we evaluate

\begin{tabular}{|c|c|c|c|c|}
\hline \multirow[b]{2}{*}{ Method } & \multicolumn{2}{|c|}{ Wikipedia } & \multicolumn{2}{|c|}{ Gigaword } \\
\hline & Sim & Rel & Sim & Rel \\
\hline \multicolumn{5}{|l|}{ Prior work } \\
\hline word2vec & 0.630 & 0.630 & 0.624 & 0.623 \\
\hline MPME & 0.506 & 0.567 & - & - \\
\hline \multicolumn{5}{|l|}{ Proposed } \\
\hline Word & 0.646 & 0.655 & 0.615 & 0.600 \\
\hline Term & 0.607 & 0.667 & 0.625 & 0.673 \\
\hline Entity & 0.594 & 0.648 & 0.634 & 0.686 \\
\hline Entity+Word & 0.718* & $0.754 *$ & $0.701 *$ & $0.722 *$ \\
\hline+ Cross & $0.697 *$ & $0.753 *$ & $0.695^{*}$ & $0.729 *$ \\
\hline
\end{tabular}

Table 4: Spearman's $\rho$ for similarity/relatedness predictions in WikiSRS, training on two corpora. All Proposed results are significantly better than MPME; *=significantly better than strongest word-level baseline $(p<0.05)$.

each type of embeddings learned in our model. Additionally, as words and entities are embedded in the same space (and thus directly comparable), we experiment with two methods of combining their information. Entity+Word sums the cosine similarities calculated between the entity embeddings and word embeddings for each pair; the Cross setting further adds comparisons of each entity in the pair to the string form of the other.

\subsubsection{Results}

Our proposed method clearly outperforms prior work and text-based baselines on both datasets. Further, we see that the words and entities learned by our model include complementary information, as combining them further increases our ranking performance by a large margin. As the results on UMNSRS could have been due to our model's ability to embed many more entities than prior methods, we also filtered the dataset to the 255 similarity pairs and 260 relatedness pairs that all evaluated entity-level methods could represent; ${ }^{4}$ Table 3 shows similar gains on this even footing. We follow Rastogi et al. (2015) in calculating significance, and use their statistics to estimate the minimum required difference for significant improvements on our datasets.

In UMNSRS, we found that cosine similarity of entities consistently reflected human judgments of similarity better than of relatedness; this reflects previous observations by Agirre et al. (2009) and Muneeb et al. (2015). Interestingly, we see the opposite behavior in WikiSRS, where relatedness is captured better than similarity in all settings. In fact, we see a number of errors of relatedness

\footnotetext{
${ }^{4}$ For WikiSRS, all methods covered all pairs.
} 


\begin{tabular}{|c|c|c|c|}
\hline Dataset & Words & Entities & Entity+Word+Cross \\
\hline \multirow{3}{*}{ UMNSRS } & Iron/Iron & Iron/Iron & Levaquin/Avelox \\
\hline & Nausea/Vomiting & Sinemet/Sinemet & Enalapril/Lisinopril \\
\hline & Lipitor/Zocor & Enalapril/Lisinopril & Carboplatin/Cisplatin \\
\hline \multirow{3}{*}{ WikiSRS } & Minas Tirith/Minas Morgul & Real Madrid/FC Barcelona & Ferrari/Lamborghini \\
\hline & Moscow/Moscow Kremlin & Minas Tirith/Minas Morgul & Moscow/Moscow Kremlin \\
\hline & Norway/Denmark & Charlize Theron/Screen Actor's Guild & Toshiro Mifune/Akira Kurosawa \\
\hline
\end{tabular}

Table 5: Top 3 pairs in the Relatedness datasets, as ranked by different embedding methods.

in WikiSRS predictions, e.g., "Hammurabi I" and "Syria" are marked highly similar, while the composers "A.R. Rahman" and "John Phillip Sousa" are marked dis-similar. MPME embeddings tend towards over-relatedness as well (e.g., ranking "Richard Feynman" and "Paris-Sorbonne University" much more highly than gold labels). Despite better similarity performance, this trend of overrelatedness also holds in biomedical embeddings: for example, C0027358 (Narcan) and C0026549 (morphine) are consistently marked highly similar across embedding methods, even though Narcan blocks the effects of opioids like morphine.

\subsubsection{Comparing entities and words}

We observe clear differences in the rankings made by entity vs word embeddings. As shown in Table 5 , highly related entities tend to have high cosine similarity, while word embeddings are more sensitive to lexical overlap and direct cooccurrence. Combining both sources often gives the most inuitive results, balancing lexical effects with relatedness. For example, while the top three pairs by combination in WikiSRS are likely to co-occur, the top three in UMNSRS are pairs of drug choices (antibiotics, ACE inhibitors, and chemotherapy drugs, respectively), only one of which is likely to be prescribed to any given patient at once.

These differences also play out in erroneous predictions. Entity embeddings often fix the worst misrankings by words: for example, "Tony Blair" and "United Kingdom" (gold rank: 28) are ranked highly unrelated (position 633) by words, but entities move this pair back up the list (position 86). However, errors made by entity embeddings are often also made by words: e.g., C0011175 (dehydration) and C0017160 (gastroenteritis) are erroneously ranked as highly unrelated by both methods. Interestingly, we find no correlation between the corpus polysemy of entity pairs and ranking performance, indicating that ambiguity of term mentions is not a significant confound for this task.

\begin{tabular}{l|ccccc} 
Method & B3 & H1 & C6 & L1 & L6 \\
\hline Words & 2.9 & 0.4 & $\mathbf{7 . 9}$ & $\mathbf{5 1 . 5}$ & $\mathbf{6 9 . 3}$ \\
Entities & $\mathbf{1 8 . 3}$ & $\mathbf{2 2 . 4}$ & 4.5 & 10.6 & 10.0 \\
\hline Oracle & 20.7 & 22.9 & 12.1 & 55.0 & 70.9
\end{tabular}

Table 6: Accuracy \% on 5 of the relations in BMASS with greatest absolute difference in word performance vs entity performance: B3 (geneencodes-product), H1 (refers-to), C6 (associatedwith), L1 (form-of), and L6 (has-free-acid-orbase-form). The better of word and entity performance is highlighted; all entity vs word differences are significant (McNemar's test; $p \ll 0.01$ ).

\subsection{Analogy completion}

We use analogy completion to further explore the properties of our joint embeddings. Given analogy $a: b:: c: d$, the task is to guess $d$ given $(a, b, c)$, typically by choosing the word or entity with highest cosine similarity to $b-a+c$ (Levy and Goldberg, 2014). We report accuracy using the top guess (ignoring $a, b$, and $c$ as candidates, per Linzen, 2016).

\subsubsection{Biomedical analogies}

To compare between word and entity representations, we use the entity-level biomedical dataset BMASS (Newman-Griffis et al., 2017), which includes both entity and string forms for each analogy. In order to test if words and entities are capturing complementary information, we also include an oracle evaluation, in which an analogy is counted as correct if either words or entities produce a correct response. ${ }^{5}$ We do not compare against prior biomedical entity embedding methods on this dataset, due to their limited vocabulary.

Table 6 contrasts the performance of different jointly-trained representations for five relations with the largest performance differences from this dataset. For gene-encodes-product and refers-to, both of which require structured domain knowledge, entity embeddings significantly

\footnotetext{
${ }^{5}$ We use the Multi-Answer setting for our evaluation (a single $(a, b, c)$ triple, but a set of correct values for $d$ ).
} 
outperform word-level representations. Many of the errors made by word embeddings in these relations are due to lexical over-sensitivity: for example, in the renaming analogy spinal epidural hematoma:epidural hemorrhage::canis familiaris: , words suggest latinate completions such as latrans and caballus, while entities capture the correct C1280551 (dog). However, on more morphological relations such as has-free-acid-orbase-form, words are by far the better option.

The success of the oracle combination method for entity and word predictions clearly indicates that not only are words and entities capturing different knowledge, but that it is complementary. In the majority of the 25 relations in BMASS, oracle results improved on words and entities alone by at least $10 \%$ relative. In some cases, as with has-freeacid-or-base-form, one method does most of the heavy lifting. In several others, including the challenging (and open-ended) associated-with, entities and words capture nearly orthogonal cases, leading to large jumps in oracle performance.

\subsubsection{General-domain analogies}

No entity-level encyclopedic analogy dataset is available, so we follow Cao et al. (2017) in evaluating the effect of joint training on words using the Google analogy set (Mikolov et al., 2013). As shown in Table 7, our Wikipedia embeddings roughly match MPME embeddings (which use annotated entity links) on the semantic portion of the dataset, but our ability to train on unannotated Gigaword boosts our results on all relations except city-in-state. ${ }^{6}$ Overall, we find that jointly-trained word embeddings split performance with wordonly skipgram training, but that word-only training tends to get consistently closer to the correct answer. This suggests that terms and entities may conflict with word-level semantic signals.

\subsection{Entity disambiguation}

Finally, to get a picture of the impact of our embedding method on downstream applications, we investigated entity disambiguation. ${ }^{7}$ Given a named entity occurrence in context, the task is to assign a canonical identifier to the entity being referred to: e.g., to mark that "New York" refers to

\footnotetext{
${ }^{6}$ We failed to precisely replicate the analogy numbers reported by Cao et al. (2017); we attribute this primarily to the different training corpus and slightly different preprocessing.

${ }^{7}$ This task is also referred to as entity linking and entity sense disambiguation.
}

\begin{tabular}{l|ccccc} 
Method & $\begin{array}{c}\text { Capital } \\
\text { (com- } \\
\text { mon) }\end{array}$ & $\begin{array}{c}\text { Capital } \\
\text { (all) }\end{array}$ & $\begin{array}{c}\text { Currency } \\
\text { City } \\
\text { in } \\
\text { State }\end{array}$ & Family \\
\hline word2vec (W) & 89.1 & 86.0 & 15.0 & $\mathbf{5 5 . 5}$ & $\mathbf{8 2 . 4}$ \\
word2vec (G) & 90.9 & 89.7 & $\mathbf{1 8 . 4}$ & 38.4 & 81.0 \\
\hline MPME (W) & 83.6 & 80.5 & 11.9 & 50.6 & 78.9 \\
\hline Proposed (W) & 90.1 & 78.7 & 9.1 & 42.5 & 75.5 \\
Proposed (G) & $\mathbf{9 2 . 7}$ & $\mathbf{9 2 . 3}$ & 16.4 & 31.3 & 81.6
\end{tabular}

Table 7: Analogy completion accuracy $\%$ on the semantic relations in the Google analogy dataset. W=Wikipedia, $\mathrm{G}=$ Gigaword.

the city in the sentence, "The mayor of New York held a press conference." It bears noting that in unambiguous cases, a terminology alone is sufficient to link the correct entity: for example, "Barack Obama" can only refer to a single entity, regardless of context. However, many entity strings (e.g., "cold", "New York") are ambiguous, necessitating the use of alternate sources of information such as our embeddings to assign the correct entity.

\subsubsection{Biomedical abstracts}

We evaluate on the MSH WSD dataset (JimenoYepes et al., 2011), a benchmark for biomedical word sense disambiguation. MSH WSD consists of mentions of 203 ambiguous terms in biomedical literature, with over 30,000 total instances. Each sample is annotated with the set of UMLS entities the term could refer to. We adopt the unsupervised method of Sabbir et al. (2016), which combines cosine similarity and projection magnitude of an entity representation $e$ to the averaged word embeddings of its contexts $C_{a v g}$ as follows:

$$
f\left(e, C_{\text {avg }}\right)=\cos \left(C_{\text {avg }}, e\right) \cdot \frac{\left\|P\left(C_{\text {avg }}, e\right)\right\|}{\|e\|}
$$

The entity maximizing this score is predicted.

We compare against concept embeddings learned by Sabbir et al. (2016). They used MetaMap (Aronson and Lang, 2010) with the disambiguation module enabled on a curated corpus of 5 million Pubmed abstracts to create a UMLS concept cooccurrence corpus for word2vec training. As shown in Table 8, our method lags behind theirs, though it clearly beats both random $(49.7 \%$ accuracy) and majority class (52\%) baselines. In addition, we leverage our jointly-embedded entities and words by adding in the definition-based model used by Pakhomov et al. (2016), which calculates an entity's embedding as the average of definitions of its neighbors in the UMLS hierarchy (McInnes et al., 2011). We use this alternate 


\begin{tabular}{l|c} 
Method & Accuracy \% \\
\hline Baselines & \\
\hline Sabbir et al. (2016) (entities; +MetaMap) & 89.3 \\
Sabbir et al. (2016) (+MetaMap, UMLS) & $\mathbf{9 2 . 2}$ \\
Pakhomov et al. (2016) (words) & 77.7 \\
\hline Proposed & 76.4 \\
\hline Entities & 80.8 \\
Definitions (joint words) & 82.7 \\
Entities+Definitions & 90.9 \\
Oracle (Entities-Definitions)
\end{tabular}

Table 8: MSH WSD disambiguation accuracy. Definitions is comparable to Pakhomov et al. (2016), using jointly-embedded words. All differences are significant (McNemar's test, $p \ll 0.01$ ).

entity embedding in Equation 4 to calculate a second score that we add to the direct entity embedding score. This yields a large performance boost of over $6 \%$ absolute, indicating that using entities and words together makes up much of the gap between our distantly supervised embeddings and the external resources used by Sabbir et al. (2016). Using the definition-based method alone with our jointly-embedded words, we see a significant increase over Pakhomov et al. (2016), indicating the benefits of joint training. However, the combined entity and definition model still yields a significantly different $2 \%$ boost in accuracy over definitions alone. Finally, we evaluate an oracle combination that reports correct if either entity or definition embeddings achieve the correct result; as shown in the last row of Table 8, this combination outperforms the entity-only method of Sabbir et al. (2016), and approaches their state-of-theart result that combines entity embeddings with a knowledge-based approach from the structure of the UMLS.

Specific errors shed more light on these differences. The definition-based method performs better in many cases where the surface form is a common word, such as coffee (68\% definition accuracy vs $28 \%$ entity accuracy) and iris (93\% definition accuracy vs $35 \%$ entity accuracy). Entities outperform on some more technical cases, such as potassium ( $74 \%$ entity accuracy vs $49 \%$ definition accuracy). Combining both approaches in the joint model recovers performance on several cases of low entity accuracy; for example, joint accuracy on coffee is $68 \%$, and on lupus (53\% entity accuracy), joint performance is $60 \%$.

\begin{tabular}{l|c} 
Method & Accuracy $\%$ \\
\hline MPME (entities; +graph structure) & $\mathbf{8 9 . 0}$ \\
\hline Wikipedia & 40.9 \\
Wikipedia + mentions & 44.6 \\
Gigaword & 58.0 \\
Gigaword + mentions & 63.9
\end{tabular}

Table 9: AIDA linking accuracy, using entity embeddings trained on Wikipedia and Gigaword. All differences are significant (McNemar's test, $p \ll$ 0.01).

\subsubsection{Newswire entities}

AIDA (Hoffart et al., 2011) is a standard dataset for entity linking in newswire, consisting of approximately 30,000 entities linked to Wikipedia page IDs. To reduce the search space, Pershina et al. (2015) provided a set of candidate entities for each mention, which we use for our experiments. The MPME model of Cao et al. (2017) achieves near state-of-the-art performance accuracy on AIDA with this candidate set, using the mention sense distributions and full document context included in the model. As our embeddings are trained without explicit entity annotations, we instead use the same cosine similarity and projection model discussed in Section 4.3.1 for this task. In contrast to our results on the biomedical data, we see performance far below the baseline on these data, as shown in Table 9.

However, we improve this performance slightly by multiplying by the similarity between the entity embedding and the average word embedding of the mention itself; this gives us roughly a further $4 \%$ accuracy for both Wikipedia and Gigaword embeddings. Using the surface form recovers several cases where entities alone yield unlikely options, e.g. Roman-era Britain instead of the United Kingdom for Britain. However, it also introduces lexical errors: for example, British in several cases refers to the United Kingdom, but the British people are often selected instead. We note that this extra score actually hurts performance on MSH WSD, where the terms are curated to be highly ambiguous, in contrast to the shorter contexts and clearer terms used in AIDA.

Two other issues bear consideration in this evaluation. Prior approaches to the AIDA dataset, including MPME, make use of the global context of entity mentions within a document to improve predictions; by using local context only, we observe some inconsistent predictions, such as selecting the cricket world cup instead of the FIFA com- 


\begin{tabular}{|c|c|c|c|c|}
\hline Entity & Words & Terms & Entities & Joint \\
\hline \multirow{3}{*}{$\begin{array}{l}\text { C0009443 } \\
\text { (common } \\
\text { cold) }\end{array}$} & k(+)-grown & & $\begin{array}{c}\text { C0041912 (upper respiratory } \\
\text { infections) }\end{array}$ & $\begin{array}{c}\text { C0041912 (upper respiratory } \\
\text { infections) }\end{array}$ \\
\hline & $\begin{array}{l}\text { legionella- } \\
\text { contaminated }\end{array}$ & short periods & C0234192 (cold sensation) & C0234192 (cold sensation) \\
\hline & & changed & $\begin{array}{c}\text { C0719425 (“Cold" } \\
\text { pharmaceutical brand) }\end{array}$ & $\begin{array}{c}\text { C0719425 ("Cold" } \\
\text { pharmaceutical brand) }\end{array}$ \\
\hline \multirow{2}{*}{$\begin{array}{l}\text { C0242797 } \\
\text { (home health } \\
\text { aides) }\end{array}$} & $\begin{array}{l}\text { homemaker- } \\
\text { home } \\
\text { voluntary- } \\
\text { sector }\end{array}$ & $\begin{array}{l}\text { home health } \\
\text { aide } \\
\text { home health } \\
\text { aides }\end{array}$ & $\begin{array}{c}\text { C1553498 (home health } \\
\text { encounter) } \\
\text { C0019855 (home care } \\
\text { services) }\end{array}$ & $\begin{array}{l}\text { home health aide } \\
\text { home health aides }\end{array}$ \\
\hline & & & $\begin{array}{l}\text { C1317851 (home health care } \\
\text { specialty) }\end{array}$ & $\begin{array}{l}\text { C1553498 (home health } \\
\text { encounter) }\end{array}$ \\
\hline
\end{tabular}

Table 10: Top 3 nearest neighbors to two UMLS entities, using words, terms, entities, or all three.

petition for world cup, in a document discussing football. Additionally, in contrast to the MSH WSD dataset, many instances in AIDA have several highly-related candidates that introduce some confusion in our results. For example, Ireland could refer to the United Kingdom of Great Britain and Ireland, the island of Ireland, or the Republic of Ireland. As our embedding training does not include gold entity links, cases like this are often errors in our predictions.

\section{Analysis of joint embeddings}

To get a more detailed picture of our joint embedding space, we investigate nearest neighbors for each point by cosine similarity. As entities in the UMLS are assigned one or more of over 120 semantic types, we first examine how intermixed these types are in our biomedical embeddings. Figure 1 shows how often an entity's nearest neighbor shares at least one semantic type with it, across the three biomedical embedding methods we evaluated. As each set of embeddings has a different vocabulary, we also restrict to the entities

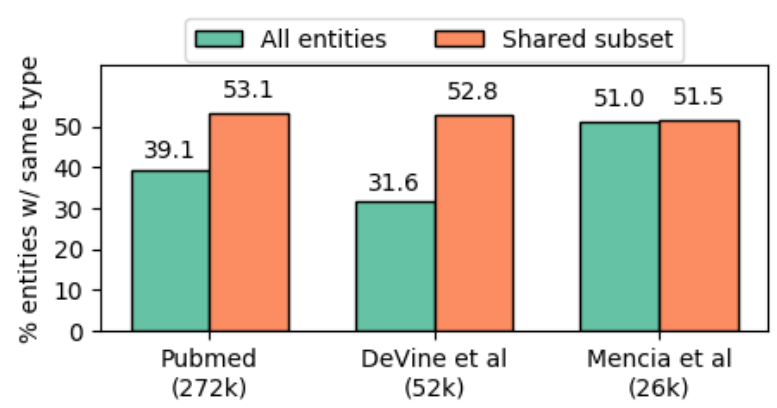

Figure 1: Percentage of UMLS entities whose nearest neighbor shares a semantic type, with no vocabulary restriction (vocab size in parentheses) and in a shared vocabulary subset. that all three can embed (approximately 11,000).

We see that our method puts entities of the same type together nearly $40 \%$ of the time, despite embedding over 270 thousand entities. On an even footing, our method puts types together significantly more often Mencia et al. (2016) (McNemar's; $p<0.05$ ), and equivalently with De Vine et al. (2014), despite using less entity-level information in training. Within our embeddings, major biological types such as bacteria, eukaryotes, mammals, and viruses all have more than $60 \%$ of neighbors with the same type, while less structured clinical types such as Clinical Attribute and Daily or Recreational Activity are in the 10-20\% range. Corpus polysemy does not appear to have any effect on this type matching (mean polysemy of 1.5 for both matched and non-matched entities).

Expanding to include the words and terms in the joint embedding space, however, we see definite qualitative effects of corpus polysemy on entity nearest neighbors. Table 10 gives nearest word, term, entity, and joint neighbors to two biomedical entities: C0009443 (the common cold; $C P=6.71$ ) and $C 0242797$ (home health aides; $C P=1$ ). For the more polysemous C0009443, where $95 \%$ of its mentions are of the word "cold" (polysemy=7), word-level neighbors are mostly nonsensical, while term neighbors are more logical, and entity neighbors reflect different senses of "cold". By contrast, the non-polysemous C0242797, which is represented by 14 different unambiguous strings, words, terms, and entities are all very clearly in line with the theme of home health aides. Notably, the common and unambiguous terms for $\mathrm{C} 0242797$ are its nearest neighbors out of all points, while only two of the top 10 neighbors to $C 0009443$ are terms. 


\section{Discussion}

Faruqui et al. (2016) observe that similarity and relatedness are not clearly distinguished in semantic embedding evaluations, and that it is unclear exactly how vector-space models should capture them. We see more evidence of this, as cosine similarity seems to be capturing a mix of the two properties in our data. This mix is clearly informative, but it empirically favors relatedness judgments, and cosine similarity is insufficient to separate the two properties.

Corpus polysemy plays a qualitative role in our embedding model, but less of a quantitative one. It does not correlate with similarity and relatedness judgments or entity disambiguation decisions, but it clearly affects the organization of the embedding space, by embedding entities with high corpus polysemy in less coherent areas than those with low polysemy. Linzen (2016) points out that for analogy completion, local neighborhood structure can interfere with standard methods; how this neighborhood structure affects predictions in more complex tasks is an open question.

Overall, we find two main advantages to our model over prior work. First, by only using a terminology and an unannotated corpus, we are able to learn entity embeddings from larger and more diverse data; for example, embeddings learned from Gigaword (which has no entity annotations) outperform embeddings learned on Wikipedia in most of our experiments. Second, by embedding entities and text into a joint space, we are able to leverage complementary information to get higher performance in both intrinsic and extrinsic tasks; an oracle model nearly matches a state-of-the-art ensemble vector and knowledge-based model for biomedical word sense disambiguation. However, our other entity disambiguation results demonstrate that there is additional entity-level information that we are not yet capturing. In particular, it is unclear whether our low performance on disambiguating newswire entities is due to a disambiguation model mismatch, a lack of information in our embeddings, or a combination of both.

\section{Conclusions}

We present a method for jointly learning embeddings of entities and text from an arbitrary unannotated corpus, using only a terminology for distant supervision. Our learned embeddings better capture both biomedical and en- cyclopedic similarity and relatedness than prior methods, and approach state-of-the-art performance for unsupervised biomedical word sense disambiguation. Furthermore, entities and words learned jointly with our model capture complementary information, and combining them improves performance in all of our evaluations. We make an implementation of our method available at github.com/OSU-slatelab/JET, along with the source code used for our evaluations and our pretrained entity embeddings. Our novel Wikipedia similarity and relatedness datasets are available at the same source.

\section{Acknowledgments}

We would like to thank Chaitanya Shivade for helpful discussions, and all of our anonymous reviewers for their invaluable advice. This research was supported in part by the Intramural Research Program of the National Institutes of Health, Clinical Research Center and through an Inter-Agency Agreement with the US Social Security Administration.

\section{References}

Eneko Agirre, Enrique Alfonseca, Keith Hall, Jana Kravalova, Marius Paca, and Aitor Soroa. 2009. A study on similarity and relatedness using distributional and WordNet-based approaches. In Proceedings of Human Language Technologies: The 2009 Annual Conference of the North American Chapter of the Association for Computational Linguistics on - NAACL '09, pages 19-27, Boulder, Colorado. Association for Computational Linguistics.

Alan R Aronson and François-Michel Lang. 2010. An overview of MetaMap: historical perspective and recent advances. Journal of the American Medical Informatics Association : JAMIA, 17(3):229-36.

Olivier Bodenreider. 2004. The Unified Medical Language System (UMLS): integrating biomedical terminology. Nucleic Acids Research, 32(90001):D267-D270.

Yixin Cao, Lifu Huang, Heng Ji, Xu Chen, and Juanzi Li. 2017. Bridge Text and Knowledge by Learning Multi-Prototype Entity Mention Embedding. In Proceedings of the 55th Annual Meeting of the Association for Computational Linguistics (Volume 1: Long Papers), pages 1623-1633, Vancouver, Canada. Association for Computational Linguistics.

Billy Chiu, Gamal Crichton, Anna Korhonen, and Sampo Pyysalo. 2016a. How to Train Good Word Embeddings for Biomedical NLP. Proceedings of the 15th Workshop on Biomedical Natural Language Processing, pages 166-174. 
Billy Chiu, Anna Korhonen, and Sampo Pyysalo. 2016b. Intrinsic Evaluation of Word Vectors Fails to Predict Extrinsic Performance. Proceedings of the 1st Workshop on Evaluating Vector Space Representations for NLP, pages 1-6.

Edward Choi, Mohammad Taha Bahadori, Andy Schuetz, Walter F Stewart, and Jimeng Sun. 2016a. RETAIN: Interpretable Predictive Model in Healthcare using Reverse Time Attention Mechanism. In NIPS, pages 1-15.

Edward Choi, Mohammad Taha Bahadori, Elizabeth Searles, Catherine Coffey, Michael Thompson, James Bost, Javier Tejedor-Sojo, and Jimeng Sun. 2016b. Multi-layer Representation Learning for Medical Concepts. Proceedings of the 22nd ACM SIGKDD International Conference on Knowledge Discovery and Data Mining - KDD '16, pages 14951504.

Youngduck Choi, Chill Yi-I Chiu, and David Sontag. 2016c. Learning Low-Dimensional Representations of Medical Concepts. In AMIA Joint Summits on Translational Science Proceedings, pages 41-50.

Lance De Vine, Guido Zuccon, Bevan Koopman, Laurianne Sitbon, and Peter Bruza. 2014. Medical semantic similarity with a neural language model. In Proceedings of the 23rd ACM International Conference on Information and Knowledge Management CIKM '14, CIKM '14, pages 1819-1822, Shanghai, China. ACM.

Wei Fang, Jianwen Zhang, Dilin Wang, Zheng Chen, and Ming Li. 2016. Entity Disambiguation by Knowledge and Text Jointly Embedding. In Proceedings of the 20th SIGNLL Conference on Computational Language Learning (CoNLL), pages 260 269. Association for Computational Linguistics.

Manaal Faruqui, Yulia Tsvetkov, Pushpendre Rastogi, and Chris Dyer. 2016. Problems With Evaluation of Word Embeddings Using Word Similarity Tasks. In Proceedings of the 1st Workshop on Evaluating Vector-Space Representations for NLP, pages 3035, Berlin, Germany. Association for Computational Linguistics.

Aditya Grover and Jure Leskovec. 2016. Node2Vec: Scalable Feature Learning for Networks. In Proceedings of the 22Nd ACM SIGKDD International Conference on Knowledge Discovery and Data Mining, KDD '16, pages 855-864, New York, NY, USA. ACM.

Johannes Hoffart, Mohamed Amir Yosef, Ilaria Bordino, Hagen Fürstenau, Manfred Pinkal, Marc Spaniol, Bilyana Taneva, Stefan Thater, and Gerhard Weikum. 2011. Robust Disambiguation of Named Entities in Text. In Proceedings of the 2011 Conference on Empirical Methods in Natural Language Processing, pages 782-792, Edinburgh, Scotland, UK. Association for Computational Linguistics.
Antonio Jimeno-Yepes and Rafael Berlanga. 2015. Knowledge based word-concept model estimation and refinement for biomedical text mining. Journal of Biomedical Informatics, 53:300-307.

Antonio J Jimeno-Yepes, Bridget $\mathrm{T}$ McInnes, and Alan R Aronson. 2011. Exploiting MeSH indexing in MEDLINE to generate a data set for word sense disambiguation. BMC Bioinformatics, 12:223.

Omer Levy and Yoav Goldberg. 2014. Linguistic Regularities in Sparse and Explicit Word Representations. In Proceedings of the Eighteenth Conference on Computational Natural Language Learning, pages 171-180, Ann Arbor, Michigan. Association for Computational Linguistics.

Tal Linzen. 2016. Issues in evaluating semantic spaces using word analogies. In Proceedings of the 1st Workshop on Evaluating Vector-Space Representations for NLP, pages 13-18.

Farzaneh Mahdisoltani, Joanna Biega, and Fabian M Suchanek. 2015. YAGO3: A Knowledge Base from Multilingual Wikipedias. In Conference on Innovative Data Systems Research (CIDR).

Bridget T McInnes, Ted Pedersen, Ying Liu, Serguei V Pakhomov, and Genevieve B Melton. 2011. Using second-order vectors in a knowledge-based method for acronym disambiguation. CoNLL 2011 - Fifteenth Conference on Computational Natural Language Learning, Proceedings of the Conference, (June):145-153.

Eneldo Loza Mencia, Gerard de Melo, and Jinseok Nam. 2016. Medical Concept Embeddings via Labeled Background Corpora. In Proceedings of the Tenth International Conference on Language Resources and Evaluation (LREC 2016), pages 46294636. European Language Resources Association (ELRA).

Tomas Mikolov, Kai Chen, Greg Corrado, and Jeffrey Dean. 2013. Efficient Estimation of Word Representations in Vector Space. arXiv preprint arXiv:1301.3781, pages 1-12.

Mike Mintz, Steven Bills, Rion Snow, and Dan Jurafsky. 2009. Distant supervision for relation extraction without labeled data. In Proceedings of the Joint Conference of the 47th Annual Meeting of the ACL and the 4th International Joint Conference on Natural Language Processing of the AFNLP, pages 1003-1011, Suntec, Singapore. Association for Computational Linguistics.

T H Muneeb, Sunil Kumar Sahu, and Ashish Anand. 2015. Evaluating distributed word representations for capturing semantics of biomedical concepts. In Proceedings of the 2015 Workshop on Biomedical Natural Language Processing (BioNLP 2015), pages 158-163, Beijing, China. Association for Computational Linguistics. 
Denis Newman-Griffis, Albert M Lai, and Eric FoslerLussier. 2017. Insights into Analogy Completion from the Biomedical Domain. In BioNLP 2017, pages 19-28, Vancouver, Canada. Association for Computational Linguistics.

Serguei Pakhomov, Bridget McInnes, Terrence Adam, Ying Liu, Ted Pedersen, and Genevieve B Melton. 2010. Semantic Similarity and Relatedness between Clinical Terms: An Experimental Study. In AMIA Annual Symposium Proceedings, pages 572-576. American Medical Informatics Association.

Serguei V S Pakhomov, Greg Finley, Reed McEwan, Yan Wang, and Genevieve B Melton. 2016. Corpus domain effects on distributional semantic modeling of medical terms. Bioinformatics, 32(August):btw529.

Robert Parker, David Graff, Junbo Kong, Ke Chen, and Kazuaki Maeda. 2011. English Gigaword Fifth Edition LDC2011T07. Linguistic Data Consortium.

Maria Pershina, Yifan He, and Ralph Grishman. 2015. Personalized Page Rank for Named Entity Disambiguation. In Proceedings of the 2015 Conference of the North American Chapter of the Association for Computational Linguistics: Human Language Technologies, pages 238-243, Denver, Colorado. Association for Computational Linguistics.

Pushpendre Rastogi, Benjamin Van Durme, and Raman Arora. 2015. Multiview LSA : Representation Learning via Generalized CCA. Proceedings of the 2015 Annual Conference of the North American Chapter of the ACL, (1961):556-566.

A. K. M. Sabbir, Antonio Jimeno Yepes, and Ramakanth Kavuluru. 2016. Knowledge-Based Biomedical Word Sense Disambiguation with Neural Concept Embeddings.

Kristina Toutanova, Danqi Chen, Patrick Pantel, Hoifung Poon, Pallavi Choudhury, and Michael Gamon. 2015. Representing Text for Joint Embedding of Text and Knowledge Bases. In Proceedings of the 2015 Conference on Empirical Methods in Natural Language Processing, pages 1499-1509, Lisbon, Portugal. Association for Computational Linguistics.

Quan Wang, Zhendong Mao, Bin Wang, and Li Guo. 2017. Knowledge Graph Embedding: A Survey of Approaches and Applications. IEEE Transactions on Knowledge and Data Engineering, 29(12):27242743.

Zhen Wang, Jianwen Zhang, Jianlin Feng, and Zheng Chen. 2014. Knowledge Graph and Text Jointly Embedding. In Proceedings of the 2014 Conference on Empirical Methods in Natural Language Processing (EMNLP), pages 1591-1601, Doha, Qatar. Association for Computational Linguistics.
Ikuya Yamada, Hiroyuki Shindo, Hideaki Takeda, and Yoshiyasu Takefuji. 2016. Joint Learning of the Embedding of Words and Entities for Named Entity Disambiguation. In Proceedings of The 20th SIGNLL Conference on Computational Natural Language Learning (CoNLL), pages 250-259, Berlin, Germany. Association for Computational Linguistics.

Zhilin Yang, William Cohen, and Ruslan Salakhudinov. 2016. Revisiting Semi-Supervised Learning with Graph Embeddings. In Proceedings of The 33rd International Conference on Machine Learning, volume 48 of Proceedings of Machine Learning Research, pages 40-48, New York, New York, USA. PMLR. 


\section{A WikiSRS construction details}

We followed a similar process to Pakhomov et al. (2010) in selecting the entity pairs to be used in our dataset. We first filtered the full list of Wikipedia pages to the subset that we learned embeddings for, and then used the entity types assigned to these pages in YAGO (Mahdisoltani et al., 2015) to restrict to only entities labeled with WordNet types organization or person, or with the YAGO type geoEntity. For each pairing of these categories (Organization-Organization, Organization-Place, Organization-Person, PlacePlace, Place-Person, and Person-Person), we manually selected 30 pairs of entities for each of the following relatedness categories: Completely Unrelated, Somewhat Unrelated, Somewhat Related, and Highly Related. These produced the list of 720 entity pairs we used for our Mechanical Turk surveys.

We augmented each survey of 30 questions with 4 manually-created validation pairs using common entities (e.g., London, New York), each of which was categorized as Highly Related or Completely Unrelated. We included these validation questions at random indices in our surveys. To evaluate if participants were reading the questions, we binned their ratings on these validation questions into 025 (Completely Unrelated), 26-50 (Somewhat Unrelated), 51-75 (Somewhat Related), and 76-100 (Highly Related). If a participant's ratings disagreed with ours on multiple validation questions, we discarded their data (we allowed disagreement on a single question, as some validation questions had high variance in responses among reliable annotators).

We recruited 6 participants for each survey, for a total of 34 unique participants across the 48 HITs. Participants were presented with a message describing the survey and stating that by clicking the button at the bottom of the message to begin the survey, they were providing informed consent to participate. Identifying participant data was not collected, and we used only the anonymous worker IDs provided by the Mechanical Turk interface to collate our data and remunerate workers. Participants were asked optional demographic questions about their age bracket and native language at the end of the survey; we did not end up using age information, but filtered our participants for those that self-reported English reading proficiency. The majority responded to a single HIT,

\begin{tabular}{c|cc|cc}
\multirow{2}{*}{ \# of raters } & \multicolumn{2}{|c|}{ Similarity } & \multicolumn{2}{c}{ Relatedness } \\
& ICC & \# pairs & ICC & \# pairs \\
\hline 4 & 0.531 & 419 & 0.467 & 180 \\
5 & 0.520 & 267 & 0.540 & 207 \\
6 & & & 0.560 & 299 \\
$>6$ & - & 2 & - & 2 \\
\hline Total & & 688 & & 688
\end{tabular}

Table 11: The intraclass correlation coefficient (ICC) among Amazon Mechanical Turk worker judgments of similarity and relatedness of pairs of Wikipedia entities. As ICC requires a fixed number of raters, but we had variable numbers of responses to each HIT, we break down the datasets by the number of workers who rated each item.

while 3 completed more than 20 . We discarded all submissions from 3 participants, as they did not report English reading proficiency (1) or did not satisfy the validation questions (2). All participants were paid state minimum wage at the time of the study for their time, regardless of whether they answered demographic questions or if we used their data in the final sample. Collection of this data was approved under Ohio State University IRB protocol 2017E0050.

To generate the final dataset, we assessed each participant's responses to the validation questions in each survey. We kept surveys for which we had at least 4 participants with satisfactory answers to the validation questions; this resulted in discarding 1 of the 24 HITs for each task. Due to 2 repeated pairs, this gave us final dataset sizes of 688 pairs for each of similarity and relatedness, 658 of which were shared between the tasks.

Following Pakhomov et al. (2010), we assessed inter-annotator agreement using the intraclass correlation coefficient (ICC). Table 11 gives the values for our datasets. The numbers reported are within the moderate range, and they correspond to the ICC numbers reported by Pakhomov et al. on the UMNSRS datasets.

The source code of our Mechanical Turk interface and data files used to generate the tasks are available at github.com/OSU-slatelab/WikisRS. 